\title{
Plasma concentrations of Granulocyte Colony- Stimulating Factor (G-CSF) in Patients with Substance Use Disorders and Comorbid Major Depressive Disorders
}

\section{Sandra Torres Galván}

Instituto de Investigación Biomédica de Málaga

María Flóres López

Instituto de Investigación Biomédica de Málaga

Pablo Romero Sanchíz

Dalhousie University

Nerea Requena Ocaña

Instituto de Investigación Biomédica de Málaga

Oscar Porras Perales

Instituto de Investigación Biomédica de Málaga

Raquel Nogueira Arjona

Dalhousie University

Fermin Mayoral

Instituto de Investigación Biomédica de Málaga

Pedro Araos

University of Malaga

Antonia Serrano

Instituto de Investigación Biomédica de Málaga

Roberto Muga

Institut d'Investigació en Ciències de la Salut Germans Trias i Pujol

Francisco J. Pavón

Instituto de Investigación Biomédica de Málaga

Nuria García Marchena ( $\sim$ ngarciam@igtp.cat)

Institut d'Investigació en Ciències de la Salut Germans Trias i Pujol

Fernando Rodriguez de Fonseca

Instituto de Investigación Biomédica de Málaga 
Keywords: G-CSF, major depressive disorders, substance use disorders

Posted Date: February 12th, 2021

DOI: https://doi.org/10.21203/rs.3.rs-152636/v1

License: (c) (1) This work is licensed under a Creative Commons Attribution 4.0 International License. Read Full License 


\section{Abstract}

Aims: Granulocyte colony-stimulating factor (G-CSF) has raised much interest due to its role to cocaine addiction in preclinical models. We analyzed the circulating expression of G-CSF in abstinent chronic users of alcohol and/or cocaine with or without comorbid major depressive disorders to investigate the role of this trophic factor with complicated substance use disorders.

Methods: We recruited 176 patients and 136 controls. Patients were divided in 50 patients with major depressive disorder (MDD) and 126 abstinent substance use disorders (SUD) patients undergoing treatments for alcohol $(\mathrm{N}=66)$ or cocaine $(\mathrm{N}=60)$ addiction according to DSM-IV-TR criteria. A blood sample was collected to examine plasma concentrations of G-CSF.

Results: The plasma concentrations of G-CSF were significantly decreased in the cocaine group compared with the SUD control group. There was a sex dimorphism in the alcohol group, with lower GCSF concentrations in women compared with men. Plasma concentrations of G-CSF were associated with abstinence and with the length of alcohol problems. The decrease in G-CSF was associated with comorbid MDD, a finding specific for SUD patients since there were no alterations of G-CSF primary settings MDD outpatients.

Conclusions: Circulating G-CSF is reduced in SUD patients, being associated to comorbid MDD. A sexdependent effect was observed in female AUD. Plasma G-CSF concentrations might be used as a predictor of length of chronic alcohol use and as a stratification role in the dual diagnosis in SUD. Further investigation is needed to explore the role of G-CSF as potential biomarker of pathogenic/prognosis in SUD population.

\section{Introduction}

Substance use disorders (SUD) are chronic neurobiological-based medical illness contributing to almost $6 \%$ of all deaths worldwide ${ }^{1}$. In the last years, it has been confirmed that SUD is associated with inflammation in the central nervous system (CNS), a process that can cause long-term behavioral alterations by activating glial cells, mainly microglia and astrocytes, and modifying the plasticity of the central nervous system ${ }^{2,3}$. Best known neuroinflammation-inducing drugs are alcohol and cocaine, and multiple studies are addressing now how these inflammatory changes contribute to the clinical course of SUD and associated pathologies ${ }^{3-6}$.

Considering alcohol, it has been described that its pharmacological actions in the CNS include multiple neurotoxic effects as activation of microglial cells, alterations in the release of several mediators such as brain derived neurotrophic factor (BDNF) ${ }^{4}$, modifications of the lipid membrane causing alteration of the cell membrane permeability, promoting changes in proliferation and maturation of neurons, and eventually neuronal apoptosis ${ }^{5-7}$. Interestingly, alcohol directly induced the activation of proinflammatory immune signaling, as revealed by the characterization of toll-like receptor 4 (TLR4) 
dependency of the glial activation responses associated with alcohol exposure ${ }^{8}$. This finding supported the inflammatory view of alcohol-induced toxic effects: preclinical studies based in reward and in alcohol withdrawal behavioral responses have revealed that there were several pro-inflammatory mediators involved in the regulation of alcohol effects ${ }^{8-11}$. As an example, the chemokine monocyte chemoattractant protein-1 (MCP-1) secreted by glial cells was found associated with alcohol consumption and alcohol-related neurodegeneration ${ }^{12,13}$. Other studies monitoring plasma concentrations of immune mediators showed alcohol-induced deregulation of certain chemokines related with immune responses such as SDF-1 [stromal derived factor (CXCL12)] and fractalkine (CX3CL1) that were found to be decreased in patients with alcohol use disorders (AUD) ${ }^{14}$. Finally, important neurotrophic factors such as BDNF and insulin-like growth factor-1 (IGF-1) were found to be profoundly affected in abstinent AUD patients ${ }^{4,15}$.

Regarding cocaine, a stimulant substance with an elevated potential of abuse contributing to a major health problem and social costs ${ }^{16}$, it is known that its consumption is associated with neuronal cytotoxicity to neurons and altered neurotrophic responses, through a nitric oxide-mediated signaling pathway ${ }^{17}$. However, despite cocaine use disorders (CUD) are mostly characterized by dysfunctions in reward-related brain circuits, deregulating mesolimbic dopaminergic reward pathways and glutamate receptor-dependent signaling cascades ${ }^{18,19}$, multiple studies also describe immune signaling alterations associated to its consumption ${ }^{8}$. Regarding circulating immunological mediators related with cocaine consumption, cytokine tumor necrosis factor alpha (TNF-a) was found to be elevated in plasma while the anti-inflammatory interleukin 10 (IL-10) was found to be decreased and linked to cocaine-related chronic stress ${ }^{20}$. Moreover, circulating concentrations of Interleukin $1 \beta(\mathrm{IL} 1 \beta)$, fractalkine and SDF-1 were found to predict severity of cocaine use disorder, suggesting a relevant contribution of the immune system to cocaine addiction ${ }^{21}$.

The contribution of the immune system is not only associated to the severity of addiction or its toxic effects, but also it might foster the development of additional co-morbid disorders. Substance use disorder patients are more likely to suffer comorbid psychiatric disorders through their lives than the general population ${ }^{22,23}$, affecting the course of the psychiatric illness ${ }^{24}$. Mood, anxiety, psychosis and personality disorders are the most prevalent diagnosis found in SUD population ${ }^{25-27}$. In this sense, immune signaling deregulations have been found to be associated with some psychiatric disorders such as major depressive disorder (MDD) ${ }^{28,29}$, something also described in SUD patients ${ }^{13}$.

The search for immune mediators participating in the natural history of addiction has brought to place new actors such as Granulocyte colony-stimulating factor (G-CSF), a glycoprotein known by its hematopoietic functions ${ }^{30}$. As a neurotrophin, its main functions include the proliferation and differentiation of myeloid progenitors ${ }^{30}$, promoting the generation of granulocyte precursors and antiapoptotic actions ${ }^{31,32}$. G-CSF receptors were studied in the CNS due to their neuroprotective properties ${ }^{33}$ including its anti-inflammatory effects found in dopaminergic neurons in the study of neurodegenerative 
disorders such as Parkinson's ${ }^{34}$ and Alzheimer disease ${ }^{35}$. Regarding addiction, G-CSF was found to be a relevant signal that promotes cocaine self-administration and consolidates cocaine seeking behavior ${ }^{36}$. However the presence of alterations of this trophic immunomodulatory factor in SUD has not been studied in humans.

The impact of addictive disorders in the health system, highlight the importance of effectives prevention programs and treatments ${ }^{37}$. Because several preclinical and clinical studies have reported an inflammatory state in SUD population, and taking on account the above described role in preclinical models of cocaine addiction, we explored the hypothesis that the circulating concentrations of the growth factor G-CSF are altered in abstinent chronic SUD patients (alcohol or cocaine users) with or without comorbid MDD. The confirmation of this hypothesis might help to further understand the contribution of immune-derived mediators to substance use disorders.

\section{Materials And Methods}

\section{Participants and recruitment}

This exploratory study is composed by 312 volunteers, including a sample of 176 patients divided in 2 cohorts, one of 50 patients with major depressive disorder (MDD) recruited from primary-care settings at Spain National Public Health System, and 125 abstinent SUD patients undergoing outpatient treatments for alcohol $(\mathrm{N}=65)$ and cocaine $(\mathrm{N}=60)$ problems. After the psychopathological evaluation the abstinent patients were further divided following the diagnosis of specific substance use disorders (SUD).

A sample of 136 healthy subjects was recruited from two different sources: a multidisciplinary staff cohort of volunteers working at the Hospital Regional Universitario de Málaga (Málaga, Spain) and a second cohort obtained from volunteers donating data and plasma to the Banco Nacional de ADN Carlos III (Salamanca, Spain). Control sample was divided in two groups [SUD control ( $N=92)$ and MDD control $(\mathrm{N}=44)]$ matching the clinical sample by age, sex and BMI ratio. Patients were recruited at the addiction treatments from Hospital Regional Universitario de Málaga (Málaga, Spain), Centro Provincial de Drogodependencias (Málaga, Spain) and Hospital Universitario 12 de Octubre (Madrid, Spain) while control participants were included from databases of healthy subjects from Hospital Regional Universitario de Málaga (Málaga, Spain).

The participation in the study was voluntary and had to meet eligibility based on inclusion criteria: both genders $\geq 18$ years old up to 65 , and the following diagnosed based on DSM-IV-TR criteria: major depressive disorder diagnosis with at least two months of depressive symptoms for the MDD group, substance use disorder diagnosis with AUD and/or CUD for the SUD group. The exclusion criteria included: personal history of long-term inflammatory diseases or cancer, severe mental disorders precluded evaluation, pregnant or breast-feeding women, at least 4 weeks of abstinence in the SUD group, any substance use disorders (except nicotine and caffeine in the MDD group and infectious diseases. 
With regard to the control groups, the inclusion criteria were being matched with the SUD or the MDD group by age and BMI ratio and having no diagnosis or medication for psychiatric disorders.

\section{Ethics statements}

Written informed consents were obtained from each participant after a complete description of the study. All the participants had the opportunity to discuss any questions or issues. The study and protocols for recruitment were approved by the Ethics Committee of the Hospital Regional Universitario de Malaga in accordance with the Ethical Principles for Medical Research Involving Human Subjects adopted in the Declaration of Helsinki by the World Medical Association (64th WMA General Assembly, Fortaleza, Brazil, October 2013) and Recommendation No. R (97) 5 of the Committee of Ministers to Member States on the Protection of Medical Data (1997), and Spanish data protection act [Regulation (EU) 2016/679 of the European Parliament and of the Council 27 April 2016 on the protection of natural persons with regard to the processing of personal data and on the free movement of such data, and repealing Directive 95/46/EC (General Data Protection Regulation). All collected data were given code numbers in order to maintain privacy and confidentiality.

\section{Clinical assessments}

All the participants in the study were evaluated using structured interviews by trained and experienced psychologists. In the SUD cohort, psychopathological disorders were diagnosed according to the DSM-IVTR criteria ${ }^{38}$ using the Spanish version of the Psychiatric Research Interview for Substance and Mental Disorders (PRISM) ${ }^{38,39}$. The PRISM is a semi-structured interview with good psychometric properties in the evaluation of substance use disorders and in the main psychiatric comorbid disorders related to substance use population ${ }^{39,40}$. In the major depression cohort the Beck Depression Inventory-II (BDI-II) was used to assess the severity of depression ${ }^{41}$. Control subjects were evaluated using the Spanish version of the Composite International Diagnostic Interview (CIDI) for the detection of psychiatric disorders ${ }^{42}$.

\section{Collection of plasma samples}

Blood extractions were conducted in the same conditions by experienced nurses in the morning after fasting for 8-12 hours. Venous blood samples were extracted into 10-ml K2 EDTA tubes (BD, Franklin Lakes, NJ, USA) and to obtain plasma, samples were centrifuged at $2200 \mathrm{~g}$ for 15 minutes $\left(4^{\circ} \mathrm{C}\right)$. Plasma was individually assayed by three rapid tests for detecting infectious diseases: HIV (Retroscreen HIV, QualPro Diagnostics-Tulip Group Ltd, Goa, India), hepatitis B (HBsAg Test, Toyo Diagnostics-Turklab Inc., Izmir, Turkey) and hepatitis C (Flaviscreen HCV, QualPro Diagnostics-Tulip Group Ltd). Infected samples were discarded following the laboratory safety protocols. Each plasma sample was registered and characterized and were stored at $80^{\circ} \mathrm{C}$ until determination.

\section{Determinations of G-CSF}

Plasma concentrations of G-CSF were determined using a selective enzyme-linked immunosorbent assay (ELISA) according to the manufacturer's instructions: human colony stimulating factor 3 granulocite 
(CSF3) ELISA Kit (\#CSB-E04563h 96T, Cusabio, Houston, Tx, USA). To perform the ELISA protocols we used $100 \mu \mathrm{L}$ of the samples into each cell, the plates were incubated 90 minutes at $37^{\circ} \mathrm{C}$. Subsequently, it was washed twice with the washing buffer, $100 \mu \mathrm{L}$ of a solution of anti-G-CSF antibodies linked with a biotin molecule were added and it was subjected to a new incubation period of 60 minutes at $37^{\circ} \mathrm{C}$. The plate was washed three times, a further $100 \mu \mathrm{L}$ of a Streptavidin-HRP containing solution in 1:1000 dilution was added, and incubated again at $37^{\circ} \mathrm{C}$ for 30 minutes. After this period of time, it was washed five times, $90 \mu \mathrm{L}$ of the TMB substrate solution in a 1:1000 dilution was added and it was incubated at $37^{\circ} \mathrm{C}$ for other 15 minutes, which was the time necessary to observe blue color in the samples and in the standard curve. At this time the reaction was stopped with a H2SO4 solution and the absorbance was measured at $450 \mathrm{~nm}$. In all cases, internal controls and calibration curve and were included in each ELISA Kit.

\section{Statistical analysis}

Data in the tables are expressed as number and percentage of subjects [N (\%)] or mean and standard deviation (SD). The significance of differences in categorical and normal continuous variables was determined using Fisher's exact test (chi-square test) and Student's $t$-test, respectively.

Analyses of covariance (ANCOVA) and analyses of variance (ANOVA) were performed to indicate the impact of the independent factors (i.e. main substances in treatment, lifetime SUD diagnosis, lifetime MDD...) controlling for additional variables as sex and age to control on the G-CSF plasma concentrations as dependent variable. The post hoc tests for multiple comparisons were performed using Tukey's correction test. The estimated marginal means [ $95 \%$ confidence intervals $(95 \% \mathrm{Cl})$ ] were described and represented in the figures. Correlation analyses were performed using the Pearson's coefficient ( $r$ ). The statistical analyses were carried out with the GraphPad Prism version 5.04 (GraphPad Software, San Diego, CA, USA), and IBM SPSS Statistical version 22 (IBM, Armonk, NY, USA). A p-value < 0.05 was considered statistically significant.

\section{Results}

\section{Socio-demographic description of the sample}

This study included 312 participants divided into SUD and MDD populations and a health group of controls matched by age and BMI with their reference group. Table 1 shows the socio-demographic variables of the sample participants. The mean age of the cocaine group was 35.4 years, and the $30 \%$ were women, with secondary education degree (76.7\%). Significant differences have been found in age between substances $(p<0.001)$; being the patients of alcohol group those who seek medical help later than the cocaine group. Moreover, were found differences between the SUD group and MDD group in the sex-balance proportion, with more women in the MDD group.

TABLE 1. Socio-demographic variables of the study groups 


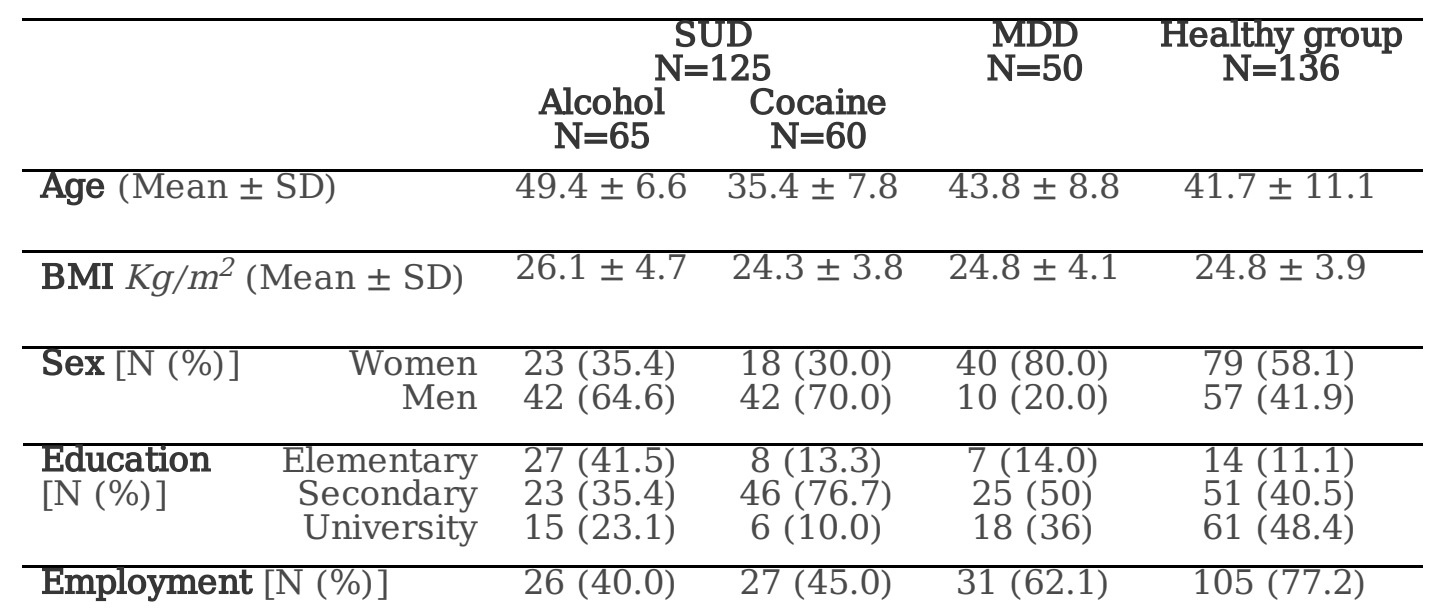

Abbreviations: $\overline{B M I=b o d y ~ m a s s}$ index; $M D D=$ major depressive disorder; $S U D=$ substance use disorder.

\section{Plasma G-CSF concentrations in relation to history of substance use disorders}

The impact of the history of substance use disorders in the plasma concentrations of G-CSF was firstly investigated using a two-way ANCOVA with the group [healthy group ( $N=92)$ and SUD $(N=125)$ ] and sex as factors and age as covariate. The multiple comparisons revealed that plasma concentration of the GCSF was significantly affected by the history of SUD $\left[F_{(1,212)}=5.915 ; p=0.016\right]$. There was a significant reduction of G-CSF plasma concentrations in the SUD group [1414.267 (95\%=1095.285-1733.248) $\mathrm{pg} / \mathrm{mL}$ ] compared with the healthy group [1999.588 (95\%Cl=1650.209-2348.967) pg/mL]. There was no effect found when the factor sex was included in the analysis, nor SUD $x$ sex interaction in the plasma concentration of G-CSF, indicating a major effect of drug consumption on the circulating levels of G-CSF. These results were represented in Figure 1A.

Because the effect found in the previous comparison, we investigate the impact of the main substances in treatment. We use a two-way ANCOVA with the group [healthy group $(\mathrm{N}=92)$; cocaine group $(\mathrm{N}=60)$ and the alcohol group $(\mathrm{N}=65)$ ] and sex as factors and age as covariate. Plasma concentration of $\mathrm{G}-\mathrm{CSF}$ was affected by the group $\left[F_{(2,210)}=3.168 ; p=0.044\right]$ with a significant reduction of G-CSF plasma concentration in the cocaine group [1256.372 (95\%Cl=768.468-1744.277) $\mathrm{pg} / \mathrm{mL}$ ] compared with the healthy group [1985.995 (95\% Cl=1641.795-2330.195)] (see Figure 1B). Interestingly, there was found an interaction effect between SUD and $\operatorname{sex}\left[F_{(2,210)}=3.393 ; p=0.035\right]$. The post hoc test revealed a significant difference $(p=0.002)$ between the G-CSF plasma concentrations in men from the alcohol group [2221.328 $(95 \% \mathrm{Cl}=1683.462-2759.193) \mathrm{pg} / \mathrm{mL}]$ compared with women from the alcohol group [1015.682 $(95 \% \mathrm{Cl}=304.437-1726.926) \mathrm{pg} / \mathrm{mL}]$ and significant differences $(\mathrm{p}=0.006)$ found between healthy women and women from the alcohol group [1994.955 $(95 \% \mathrm{Cl}=2488.334-1501.575) \mathrm{pg} / \mathrm{mL}$ and 1015.682 $(95 \% \mathrm{Cl}=304.437-1726.926)$, respectively]. These results were represented in Figure 1C.

Interestingly, when we compare with the patients with both substance use disorders (alcohol and cocaine), the effects seen above lost their significance, suggesting that the combination of both drugs abolished the differences observed in primary CUD/AUD patients. We use a two-way ANCOVA with the group [healthy group ( $\mathrm{N}=92)$; CUD group ( $\mathrm{N}=51)$; AUD group ( $\mathrm{N}=50)$ and PoliUD group $(\mathrm{N}=24)$ ] and sex as 
factors and age as covariate. There were no effects found by the group neither the interaction between group and sex. In Supplementary S1 were represented the estimated marginal means of the plasma GCSF concentrations based in the history of substance use disorders.

\section{Plasma G-CSF concentrations in relation to substance-use characteristics}

Since there were found differences on G-CSF plasma concentration according to the history of substance use disorder (either alcohol or cocaine), we explore additional relevant variables related to the specific disorder group, such as severity, length of abstinence or years of SUD diagnosis. To this end SUD patients were divided according to alcohol or cocaine group for correlation analysis (Table 2). At the time of the evaluation, the alcohol group had a mean of 7.8 DSM-IV-TR alcohol disorder criteria and an average length of 160 days of abstinence with a positive correlation of G-CSF plasma concentration with the length of alcohol abstinence. The cocaine group had a mean of 7.6 DSM-IV-TR cocaine disorder criteria and an average of 55.2 days of abstinence at the moment of the evaluation. We did not found any correlation between CUD-related variables and the G-CSF plasma concentrations in the CUD group.

TABLE 2. Correlation between G-CSF concentrations and substance-use variables

\begin{tabular}{lcccc}
\hline \multicolumn{1}{c}{ Variables } & \multicolumn{2}{c}{$\begin{array}{c}\text { Alcohol } \\
\mathbf{N}=65\end{array}$} & \multicolumn{2}{c}{$\begin{array}{c}\text { Cocaine } \\
\mathbf{N}=60\end{array}$} \\
\cline { 2 - 5 } & rho & $p$-value & rho & $p$-value \\
\hline DSM-IV-TR criteria [1-11] & -0.092 & 0.466 & -0.239 & 0.066 \\
& & & & \\
\hline Length of abstinence & 0.283 & $\mathbf{0 . 0 2 2}$ & 0.032 & 0.813 \\
& & & & \\
\hline Years of SUD diagnosis & 0.127 & 0.314 & -0.076 & 0.518
\end{tabular}

Bold values are statistically significant for $p<0.05$ after Spearman's correlation.

\section{Plasma G-CSF concentrations in relation to psychiatric disorders}

Since SUD is often associated with psychiatric co-morbidity, we analyzed whether G-CSF concentrations might be different regarding the presence of psychopathology. Our SUD sample was characterized to display an elevated prevalence of psychiatric disorders. The $51.6 \%$ of the SUD sample has other mental comorbid diagnosis, being the $31.7 \%$ mood disorders, $27 \%$ anxiety disorders, $15 \%$ cluster B personality disorders and $4.8 \%$ psychotic disorders, the most prevalent. No statistical differences found in the sex proportion between them. In Table 3, was described the clinical comorbid characteristics according to alcohol and cocaine group.

TABLE 3. Clinical characteristics of the SUD group 


\begin{tabular}{|c|c|c|c|}
\hline \multirow[t]{2}{*}{$\begin{array}{l}\text { Psychopathological evaluation } \\
{[\mathrm{N}(\%)]}\end{array}$} & $\begin{array}{l}\text { Alcohol } \\
\mathrm{N}=65\end{array}$ & $\begin{array}{c}\text { Cocaine } \\
\mathrm{N}=60\end{array}$ & \multirow[t]{2}{*}{$p$-value } \\
\hline & {$[\mathrm{N}(\%)]$} & {$[\mathrm{N}(\%)]$} & \\
\hline Major depressive disorder (MDD) & $29(44.6)$ & $11(18.3)$ & 0.002 \\
\hline Dysthymia disorder & $2(3.1)$ & $3(5.0)$ & 0.672 \\
\hline Cyclothymic disorder & $1(1.5)$ & $1(1.7)$ & - \\
\hline Schizophreniform disorder & - & $1(1.7)$ & 0.480 \\
\hline Psychotic disorder not specified & $5(7.7)$ & $1(1.7)$ & 0.210 \\
\hline Social phobia & $2(3.1)$ & $3(5.0)$ & 0.670 \\
\hline Panic disorder & $10(15.4)$ & $2(3.3)$ & 0.032 \\
\hline Agoraphobia disorder & $1(1.5)$ & $2(3.3)$ & 0.670 \\
\hline Generalized anxiety disorders & $3(4.6)$ & $2(3.3)$ & 1.000 \\
\hline Obsessive-compulsive disorder & $1(1.5)$ & $6(10.0)$ & 0.054 \\
\hline Post-traumatic stress disorder (PTSD) & $7(10.8)$ & $6(10.0)$ & 1.000 \\
\hline Anorexia disorder & $1(1.5)$ & $10(16.7)$ & 0.003 \\
\hline Bulimia disorder & $3(4.6)$ & $16(26.7)$ & 0.001 \\
\hline Antisocial personality disorder & $3(4.6)$ & $2(3.3)$ & 1.000 \\
\hline Borderline personality disorder & $10(15.4)$ & $5(8.3)$ & 0.277 \\
\hline Attention deficit hyperactivity disorder & $6(10.2)$ & $10(16.7)$ & 0.421 \\
\hline
\end{tabular}

As shown in the psychopathological description of the SUD sample, we examined the effect of the most prevalent disorders in our abstinent SUD sample in the G-CSF plasma concentrations using two-way ANCOVAs.

The two-way ANCOVA showed a main effect in the diagnosis of MDD in the abstinent SUD patients, but there were no other significant effects found in the G-CF plasma concentrations in the other comorbid diagnosis according to the psychopathological evaluation (see Supplementary S2).

Because the $66.7 \%$ of the abstinent SUD group was using psychoactive medication during the last 12 months: antidepressants (40.7\%), anxiolytics (40.7\%), disulfiram (39\%), anticraving (22.8\%), and antipsychotics (8.9\%), respectively, we monitored the effect of this medication on G-CSF levels. The 
analysis revealed no effect of medication in the G-CSF plasma concentrations. The differences in G-CSF concentrations based on the psychiatric medication of the SUD sample are described in Table 4.

TABLE 4. Plasma G-CSF concentrations in the SUD group according to psychiatric medication

\begin{tabular}{|c|c|c|c|c|c|}
\hline \multirow[t]{3}{*}{ Variables } & \multicolumn{2}{|c|}{ SUD N=125 } & \multicolumn{3}{|c|}{ Statistics ${ }^{(1)}$} \\
\hline & Non medicated & Medicated & F & $\mathrm{df}$ & $p$-value \\
\hline & $\begin{array}{l}\text { Mean } \\
95 \% \mathrm{CI}\end{array}$ & $\begin{array}{l}\text { Mean } \\
95 \% \mathrm{CI}\end{array}$ & & & \\
\hline Antidepressants & $\begin{array}{c}1566.51 \\
{[1173.88-1959.14]}\end{array}$ & $\begin{array}{c}1265.11 \\
{[790.70-1739.53]}\end{array}$ & 0.939 & 1,121 & 0.335 \\
\hline Anxiolytics & $\begin{array}{c}1495.95 \\
{[1102.07-1889.83]}\end{array}$ & $\begin{array}{c}1368.13 \\
{[892.21-1844.05]}\end{array}$ & 0.168 & 1,121 & 0.683 \\
\hline Anticonvulsants & $\begin{array}{c}1426.41 \\
{[1080.97-1771.86]}\end{array}$ & $\begin{array}{c}1503.63 \\
{[867.33-2139.94]}\end{array}$ & 0.045 & 1,121 & 0.833 \\
\hline Antipsychotics & $\begin{array}{c}1359.36 \\
{[1045.25-1673.47]}\end{array}$ & $\begin{array}{c}2305.69 \\
{[1303.40-3307.97]}\end{array}$ & 3.182 & 1,121 & 0.077 \\
\hline Disulfiram & $\begin{array}{c}1418.50 \\
{[1029.71-1807.29]}\end{array}$ & $\begin{array}{c}1483.82 \\
\text { [997.83-1969.81] }\end{array}$ & 0.043 & 1,121 & 0.836 \\
\hline
\end{tabular}

(1) Data were analyzed by ANOVA and ${ }^{*} p<0.05$ denote a significant main effect of group factor.

Plasma G-CSF concentrations in relation to comorbid MDD in substance use disorders

As indicated in the previous section, the association between the presence of comorbid MDD in abstinent SUD patients and G-CSF plasma concentrations was investigated using a two-way ANCOVA with the group [SUD with MDD $(\mathrm{N}=40)$ and SUD without MDD $(\mathrm{N}=85)$ ] and sex as factors and controlled for age. We found a clear effect of MDD diagnosis on circulating G-CSF concentrations $\left[\mathrm{F}_{(1,120)}=6.568 ; p=0.012\right]$ with a significant decrease in SUD patients with MDD diagnosis [833.727 (95\% Cl=289.896-1377.558) $\mathrm{pg} / \mathrm{mL}]$ compared with SUD group with no comorbid MDD [1722.798 $(95 \% \mathrm{Cl}=1327.901-2117.695)$ $\mathrm{pg} / \mathrm{mL}]$. Interestingly, an interaction effect between MDD and $\operatorname{sex}\left[F_{(1,120)}=4.055 ; p=0.046\right]$ was observed. The post hoc corrected Tukey test revealed a significant difference $(p=0.017)$ between the GCSF plasma concentrations in no comorbid MDD women compared with women with comorbid MDD.

SUD patients diagnosed with MDD were divided according to DSM-IV-TR criteria into primary and substance-induced MDD. The impact of the type of comorbid MDD in SUD patients was studied using a one-way ANOVA but there were not found statistical differences in the plasma concentrations of the neurotrophin G-CSF (see Table 5) regarding the origin of the MDD diagnosis.

TABLE 5. Plasma G-CSF concentrations in the SUD group according to the type of comorbid depression 


\begin{tabular}{|c|c|c|c|c|c|c|c|}
\hline & \multicolumn{4}{|c|}{$\begin{array}{c}\text { SUD sample } \\
(\mathbf{N}=125)\end{array}$} & \multicolumn{3}{|c|}{ Statistics ${ }^{(1)}$} \\
\hline & $\begin{array}{l}\text { No MDD } \\
(\mathrm{N}=85)\end{array}$ & $\begin{array}{c}\text { Primary } \\
\text { MDD } \\
(\mathrm{N}=15)\end{array}$ & $\begin{array}{c}\text { Induced } \\
\text { MDD } \\
(\mathrm{N}=16)\end{array}$ & $\begin{array}{l}\text { Both MDD } \\
(\mathrm{N}=9)\end{array}$ & $\mathrm{F}$ & df & $\begin{array}{c}p- \\
\text { value }\end{array}$ \\
\hline & $\begin{array}{l}\text { mean } \\
95 \% \mathrm{CI}\end{array}$ & $\begin{array}{l}\text { mean } \\
95 \% \mathrm{CI}\end{array}$ & $\begin{array}{l}\text { mean } \\
95 \% \mathrm{CI}\end{array}$ & $\begin{array}{l}\text { mean } \\
95 \% \mathrm{CI}\end{array}$ & & & \\
\hline $\begin{array}{l}\text { G-CSF plasma } \\
\text { concentrations }\end{array}$ & $\begin{array}{c}1715.51 \\
{[1356.89-} \\
2074.12]\end{array}$ & $\begin{array}{c}808.19 \\
{[-45.49-} \\
1661.83]\end{array}$ & $\begin{array}{l}1358.99 \\
{[532.45-} \\
2185.55]\end{array}$ & $\begin{array}{c}517.69 \\
{[-584.38-} \\
1619.76]\end{array}$ & 2.396 & 3,121 & 0.072 \\
\hline
\end{tabular}

(1) Data were analyzed by ANOVA and ${ }^{*} p<0.05$ denote a significant main effect of group factor.

Moreover, to investigate the impact of comorbid MDD in the different groups we separate the sample by the main substance in treatment. Regarding the alcohol group, a one-way ANOVA with the group [MDD $(\mathrm{N}=29) ;$ no MDD ( $\mathrm{N}=36)$ ] showed that plasma concentration of $\mathrm{G}-\mathrm{CSF}$ was affected by the MDD diagnosis $\left[F_{(1,63)}=4.327 ; p=0.042\right]$ with a reduction of G-CSF plasma concentration found in alcohol patients with comorbid MDD [1008.093 $(95 \% \mathrm{Cl}=344.885-1671.301) \mathrm{pg} / \mathrm{mL}]$ compared with alcohol with no comorbid MDD [1935.686 $(95 \% \mathrm{Cl}=1340.438-2530.933) \mathrm{pg} / \mathrm{mL}]$. These results were represented in Figure 2. In contrast, in the cocaine group there were not found differences in the plasma concentrations of G-CSF in the comorbid MDD diagnosis.

Finally, we analyzed if the plasma concentrations of G-CSF were affected by the current psychiatric medication in the alcohol group, but the analyses did not showed any statistical differences in the G-CSF levels in the alcohol patients receiving medication (see Supplementary S3).

\section{Plasma G-CSF concentrations in relation to major depressive disorders}

Because there was some evidence that peripheral alterations of this glycoprotein could be related with the presence of comorbid MDD in SUD population, we investigated the plasma concentrations of G-CSF in primary-care patients diagnosed with depression (MDD) with no substance use disorders. A two-way ANCOVA was performed with the group [Healthy group ( $N=44)$ and MDD group $(N=50)$ ] and sex as factors and controlled for age. We did not found any significant effects from group, age, sex, or either the interaction between factors (Figure 3A), suggesting that is the concurrence of SUD + MDD the origin of the specific decrease in circulating levels of this immunomodulatory trophic factor.

The MDD group was using psychiatric medication during their treatment, mostly antidepressants (36\%) and anxiolytics (40\%). Moreover, and based in recent research in our group that found $\mathrm{n}$ acylethanolamide levels elevated in MDD patients with antidepressant treatment medication ${ }^{43}$, we decided to investigate the possible effects of antidepressant and anxiolytic medication in the primarycare patients diagnosed with depression (MDD) with no substance use disorders. The analysis revealed no significant effect based on the current psychiatric medication treatment in G-CSF plasma concentrations. The marginal means were represented in Figure 3B.

\section{Discussion}


In the present study, we examined the granulocyte colony-stimulating factor (G-CSF) in the peripheral plasma concentrations of abstinent patients with alcohol or cocaine use disorders and in a cohort of major depressive disorder patients, who were recruited from active programs in treatment primary-care settings. Data were compared with healthy controls. Additionally, all these participants were characterized through psychopathological assessment based on the DSM-IV-TR criteria. The main findings of this study suggest that: 1) The plasma concentrations of G-CSF was significantly decreased in patients requesting treatment for cocaine use disorders, compared with the SUD control population of healthy people; 2) A history of AUD also affected G-CSF. In this case, there was a sexual dimorphism in the plasma concentrations of G-CSF in the alcohol group, with lower plasma levels in women compared with men; 3) The plasma concentrations of G-CSF were associated with the length of abstinence in the SUD groups; 4) The plasma concentrations of G-CSF were associated with the age, the length of abstinence and the years of diagnosis in the alcohol group; 5) G-CSF plasma concentrations were further decreased in the AUD patients with comorbid MDD disorders suggesting a possible potential role of this immunotrophic factor in dual diagnosis rather than in primary depression disorders.

The present results confirm that in addition to its known role as a modulator of myeloid cells from the bone marrow, G-CSF might contribute to the history of addiction in both AUD and CUD patients, although the nature of this contribution demands further research. While in animals this immunotrophic signal helps to consolidate cocaine reward/cocaine seeking behavior, in abstinent humans we observed that GCSF concentrations are decreased in SUD, with an almost significant negative correlation with the severity of CUD. In the absence of data on acute cocaine effects on circulating G-CSF, we can only speculate on the nature of this finding. If the role of this factor in preclinical models of cocaine addiction is to boost/sustain cocaine seeking behavior ${ }^{36}$, it is reasonable to think that after prolonged exposure to cocaine and abstinence, this factor might be down regulated in severe addicted patients, following the allostatic model set in place for most of the biological modulatory transmitters on addiction ${ }^{44}$. This finding demands to be conclusively determined. Regarding alcohol, we do not have information on the role of G-CSF in animal models of alcohol addiction, but our results clearly support that this immunotrophic factor might contribute to alcohol abuse and dependence, as it does with cocaine. Furthermore, this analyte has also been studied related to memory functions in rats, showing that the deficiency of G-CSF concentrations in hippocampus decreased spatial learning performance and memory formations ${ }^{45}$. Therefore, we consider that it would be interesting to measure the role of this growth factor in a neuropsychological cohort of patients with substance use disorders and substance-induced memory deficits. This is especially relevant in the case of alcohol, since this drug has been linked clearly to memory deficits and deceased circulating neurotrophic factors.

Another important aspect of the present study is the existence of sex-related differences in circulating GCSF, a fact of interest for gender-specific stratification and interventions in addiction. The sex is an important physiological factor studied as an underlying susceptibility, with differential outcomes and treatment in biomedical research ${ }^{46,47}$. The literature describe sexual dimorphism in many alcohol-derived consequences, either psychiatric disturbances and injuries in the organ system ${ }^{48}$; suggesting that 
women are more sensitive to alcohol behavioral outcomes whereas men may be more sensitive to the alcohol neurophysiologic effects ${ }^{49}$. Moreover, considering the variables related with the alcohol use disorder diagnosis, in our study we did not found differences in the length of AUD diagnosis neither less duration of alcohol use in women but in the number of abstinence periods achieved after the years. This result is in accordance with previous studies searching sex differences reporting that lifetime prolonged alcohol abstention is more common among women, although this difference is strongly influenced by socio-cultural factors ${ }^{50}$.

Regarding the differences found in the growth factor G-CSF attributable to the interaction of the sex and the alcohol use disorders; we observe a sexual dimorphism response, with decreased G-CSF plasma concentration in female AUD patients. Accordingly to these sex differentially affected results, other preclinical studies have found sex-differences in the neuro-regulation, microglia signaling, reward processes and homeostasis in brain development ${ }^{51,52}$. There were found sexual dimorphism in proinflammatory mediators in chronic alcohol consumption; with a differential action in the immune system and a marked suppression of immune signaling in males in corticosterone response, concretely in transforming growth factor $\beta$-1 (TGF $\beta-1$ ) and interleukin 6 (IL-6) ${ }^{53}$. Moreover, other cytokines as interleunkin-1 $\beta$ (IL-1 $\beta)$ and tumor necrosis factor $a(\mathrm{TNF}-\mathrm{a})$ were found up-regulated in females mice compared with males ${ }^{54}$. In the same way, studies in SUD population found differences between sexes in circulating factors, such $C$-reactive protein in the alcohol consumption ${ }^{55}$. Regarding the cocaine use, concentrations of G-CSF were affected by the lifetime CUD, although the sexual dimorphism has not been clearly found between them. Moreover, the absence of sexual differences could be supported by previous clinical studies indicating that chronic cocaine intake could attenuate sex-differences in the immune system expression ${ }^{56}$.

On the other hand, epidemiological studies showed a bidirectional relation between SUD and comorbid disorders with genetic vulnerability as a common etiology factor ${ }^{57,58}$ and sex differences observed in the prevalence of psychiatric comorbid disorders in SUD population ${ }^{59}$. Despite the elevated rates of comorbid mood and anxiety disorders found in our sample, we did not found differences between men and women. However, follow-up studies pointed that there were sex-differences in the course of comorbid affective disorders in AUD population, with more therapeutic need and a worse prognosis in short-term outcomes in male than female patients ${ }^{60}$.

We demonstrated a positive correlation of plasma concentration of G-CSF with the length of alcohol abstinence and the years of diagnosis problems. As in the case of the growth factor G-CSF, other regulated markers were found related with the length of alcohol abstinence as the fatty acid derivative oleoilethanolamide (OEA). OEA is a satiety factor regulated downward as the length of abstinence increase in patients with alcohol use disorders acting as a marker of abstinence ${ }^{61}$; while G-CSF seems to behave as a possible predictor of chronic alcohol use. 
Finally, our results showed that G-CSF plasma concentrations were decreased in abstinent AUD patients with comorbid MDD disorders. There are long-lasting stress effects studied in the development of mood disorders ${ }^{62}$ with several deregulations produced on the pro-inflammatory status ${ }^{63}$. Recent studies in our group confirmed differences found in other peripheral mediators related to the presence of comorbid psychiatric disorders in AUD population; the circulating levels of the chemokine eotaxin-1 (CCL11) were found decreased in AUD patients with mood and anxiety comorbid disorders ${ }^{14}$; and additionally, other peripheral plasma levels of acyl-glycerol concentrations [2-araquidonil-glycerol (2-AG) and 2-linoleoylglycerol (2-OG)] were found decreased in AUD patients with comorbid anxiety disorders ${ }^{26}$.

\section{Conclusions and limitations of the present study}

These findings support the importance of monitoring G-CSF in the context of substance use disorders and psychiatric comorbidity, but we are aware of the limitations of this study: 1) There are uncontrolled social variables from the patients SUD cohorts that can be considered as a source of variability. It is important to replicate these findings in other cohorts from different geographical and cultural backgrounds. 2) There are statistical limitations related to parametric assumptions, the sample size and the number of independent variables in the linear models that precluded the inclusion of more covariates (principally socio-demographic variables); 3 ) Larger samples of female substance use disorders patients and additional experimental groups should be included in further studies to reproduce these results and include more relevant covariates; 4) Longitudinal studies are also needed to monitor changes in this growth factor during acute exposure, active consumption and along abstinence if possible in the same SUD patients.

In conclusion, these findings support an effect of substance use disorders in G-CSF plasma circulating concentrations, with a significant decrease in the CUD sample and a sexual dimorphism in the plasma concentrations of G-CSF in abstinent AUD patients with lower levels in women. Interestingly, we found decreased plasma concentrations of G-CSF associated with comorbid lifetime major depressive disorders diagnosis in AUD patients. Conversely, these findings in comorbid MDD were not observed in abstinent CUD patients. Additionally, the plasma concentrations of G-CSF correlated with alcohol related variables as the length of abstinence, an interesting variable related with a good prognosis of the substance use disorders. Moreover, the last part of the study in patients with MDD demonstrated that the deregulation of G-CSF plasma concentration was related with the combination of AUD and comorbid MDD rather than in primary MDD. Further research is necessary to elucidate the role of G-CSF as a potential biological marker of neurotrophic state in dual diagnosis.

\section{Declarations}

\section{Funding}

The present study has been supported by the following programs and research projects: Subprograma Redes Temáticas RETICS (Red de Trastornos Adictivos RD12/0028/0021, RD16/0017/001 and, 
RD17/0017/0003) funded by Instituto de Salud Carlos III (ISCIII), Ministerio de Economía y Competitividad (MINECO) and the European Regional Development Funds/European Social Fund (ERDF/ESF); Proyectos de Investigación en Salud (PI16/01698, PI17/02026, PI19/01577, PI20/0883) funded by ISCIII and ERDF/ESF; Proyectos de Investigación en Drogodependencias (PND2017/043, PND2018/033, PND2018/044 and PND2019/040) funded by Delegación del Gobierno para el Plan Nacional sobre Drogas, Ministerio de Sanidad, Secretaría de Estado de Sanidad and ERDF/ESF; Proyecto de Investigación en Salud (PI-0140-2018) funded by Consejería de Salud y Bienestar Social, Junta de Andalucía and ERDF/ESF. NGM holds a "Sara Borrell" research contract (CD19/00019) funded by ISCIII and ERDF/ESF. FJP and AS hold a "Miguel Servet II" research contract (CPII19/00022, CPII19/00031 respectively) funded by ISCIII and ERDF/ESF.

\section{Acknowledgements}

The authors are grateful to G. Rubio, J.J. Ruiz, C. Sánchez and M. Soria, for their valuable assistance throughout the clinical part of the study.

\section{Conflict of interest declaration}

None

\section{References}

1. WHO. Global status report on alcohol and health on alcohol and health. (World Health Organization).

2. Lawson, L. J., Perry, V. H., Dri, P. \& Gordon, S. Heterogeneity in the distribution and morphology of microglia in the normal adult mouse brain. Neuroscience.39, 151-170 (1990).

3. Lacagnina, M. J., Rivera, P. D. \& Bilbo, S. D. Glial and neuroimmune mechanisms as critical modulators of drug use and abuse. Neuropsychopharmacology: official publication of the American College of Neuropsychopharmacology.42, 156-177 (2017).

4. Garcia-Marchena, N. et al. Decreased plasma concentrations of BDNF and IGF-1 in abstinent patients with alcohol use disorders. PLoS One.12, e0187634 https://doi.org/10.1371/journal.pone.0187634 (2017).

5. Weiss, F. \& Porrino, L. J. Behavioral neurobiology of alcohol addiction: recent advances and challenges. Journal of Neuroscience.22, 3332-3337 (2002).

6. Nixon, K. \& Crews, F. T. Temporally specific burst in cell proliferation increases hippocampal neurogenesis in protracted abstinence from alcohol. Journal of Neuroscience.24, 9714-9722 (2004).

7. Kohno, M. et al. Neuroinflammation in addiction: A review of neuroimaging studies and potential immunotherapies. Pharmacology Biochemistry and Behavior(2019).

8. Coller, J. K. \& Hutchinson, M. R. Implications of central immune signaling caused by drugs of abuse: mechanisms, mediators and new therapeutic approaches for prediction and treatment of drug dependence. Pharmacology \& therapeutics.134, 219-245 (2012). 
9. Kim, Y. K. et al. Increased transforming growth factor-beta1 in alcohol dependence. Journal of Korean medical science.24, 941-944 https://doi.org/10.3346/jkms.2009.24.5.941 (2009).

10. Gonzalez-Quintela, A. et al. Influence of acute alcohol intake and alcohol withdrawal on circulating levels of IL-6, IL-8, IL-10 and IL-12. Cytokine.12, 1437-1440 https://doi.org/10.1006/cyto.2000.0715 (2000).

11. Heberlein, A. et al. BDNF and GDNF serum levels in alcohol-dependent patients during withdrawal. Progress in neuro-psychopharmacology \& biological psychiatry.34, 1060-1064 https://doi.org/10.1016/j.pnpbp.2010.05.025 (2010).

12. He, J., Crews, F. T. \& Increased MCP-1 and microglia in various regions of the human alcoholic brain. Experimental neurology.210, 349-358 (2008).

13. Blednov, Y. A. et al. Perturbation of chemokine networks by gene deletion alters the reinforcing actions of ethanol. Behavioural brain research.165, 110-125 (2005).

14. Garcia-Marchena, N. et al. Plasma Chemokines in Patients with Alcohol Use Disorders: Association of CCL11 (Eotaxin-1) with Psychiatric Comorbidity. Front Psychiatry.7, 214 https://doi.org/10.3389/fpsyt.2016.00214 (2016).

15. Silva-Pena, D. et al. Alcohol-induced cognitive deficits are associated with decreased circulating levels of the neurotrophin BDNF in humans and rats. Addict Biol.24, 1019-1033 https://doi.org/10.1111/adb.12668 (2019).

16. Degenhardt, L. et al. The global epidemiology and burden of opioid dependence: Results from the Global Burden of Disease 2010 study. Vol. 109 (2014).

17. Guha, P., Harraz, M. M. \& Snyder, S. H. Cocaine elicits autophagic cytotoxicity via a nitric oxideGAPDH signaling cascade. Proceedings of the National Academy of Sciences of the United States of America.113, 1417-1422 https://doi.org/10.1073/pnas.1524860113 (2016).

18. Siciliano, C. A., Fordahl, S. C. \& Jones, S. R. Cocaine Self-Administration Produces Long-Lasting Alterations in Dopamine Transporter Responses to Cocaine. The Journal of neuroscience: the official journal of the Society for Neuroscience.36, 7807-7816 https://doi.org/10.1523/jneurosci.465215.2016 (2016).

19. Wolf, M. E. Synaptic mechanisms underlying persistent cocaine craving. Nature reviews. Neuroscience.17, 351-365 https://doi.org/10.1038/nrn.2016.39 (2016).

20. Fox, H. C. et al. Immune system inflammation in cocaine dependent individuals: implications for medications development. Human psychopharmacology.27, 156-166 https://doi.org/10.1002/hup.1251 (2012).

21. Araos, P. et al. Plasma profile of pro-inflammatory cytokines and chemokines in cocaine users under outpatient treatment: influence of cocaine symptom severity and psychiatric co-morbidity. Addict Biol.20, 756-772 https://doi.org/10.1111/adb.12156 (2015).

22. Torrens, M., Mestre-Pinto, J. I., Montanari, L., Vicente, J. \& Domingo-Salvany, A. Patología dual: una perspectiva europea Dual diagnosis: an European perspective. Adicciones.29, 3-5 (2017). 
23. Hasin, D. S., Stinson, F. S., Ogburn, E. \& Grant, B. F. Prevalence, correlates, disability, and comorbidity of DSM-IV alcohol abuse and dependence in the United States: results from the National Epidemiologic Survey on Alcohol and Related Conditions. Arch Gen Psychiatry.64, 830-842 https://doi.org/10.1001/archpsyc.64.7.830 (2007).

24. Kessler, R. C. The epidemiology of dual diagnosis. Biological psychiatry.56, 730-737 (2004).

25. Pettinati, H. M., O'Brien, C. P. \& Dundon, W. D. Current status of co-occurring mood and substance use disorders: a new therapeutic target. American Journal of Psychiatry.170, 23-30 (2013).

26. Garcia Marchena, N. et al. Psychiatric comorbidity and plasma levels of 2-acyl-glycerols in outpatient treatment alcohol users. Analysis of gender differences. Adicciones.29, 83-96 https://doi.org/10.20882/adicciones.728 (2016).

27. Araos, P. et al. Differences in the Rates of Drug Polyconsumption and Psychiatric Comorbidity among Patients with Cocaine Use Disorders According to the Mental Health Service. J Psychoactive Drugs.49, 306-315 https://doi.org/10.1080/02791072.2017.1342151 (2017).

28. Setiawan, E. et al. Role of translocator protein density, a marker of neuroinflammation, in the brain during major depressive episodes. JAMA psychiatry.72, 268-275 (2015).

29. Bhattacharya, A., Derecki, N. C., Lovenberg, T. W. \& Drevets, W. C. Role of neuro-immunological factors in the pathophysiology of mood disorders. Psychopharmacology.233, 1623-1636 (2016).

30. Welte, K. et al. in Modern Trends in Human Leukemia VI New Results in Clinical and Biological Research Including Pediatric Oncology 398-401 (Springer, 1985).

31. Lee, S. T. et al. Granulocyte colony-stimulating factor enhances angiogenesis after focal cerebral ischemia. Brain research.1058, 120-128 (2005).

32. Metcalf, D. et al. Biologic properties in vitro of a recombinant human granulocyte-macrophage colony-stimulating factor. (1986).

33. Schneider, A. et al. The hematopoietic factor G-CSF is a neuronal ligand that counteracts programmed cell death and drives neurogenesis. The Journal of clinical investigation.115, 20832098 (2005).

34. Meuer, K. et al. Granulocyte-colony stimulating factor is neuroprotective in a model of Parkinson's disease. Journal of neurochemistry. 97, 675-686 (2006).

35. Tsai, K. J., Tsai, Y. C. \& Shen, C. K. J. G-CSF rescues the memory impairment of animal models of Alzheimer's disease. The Journal of experimental medicine.204, 1273-1280 (2007).

36. Calipari, E. S. et al. Granulocyte-colony stimulating factor controls neural and behavioral plasticity in response to cocaine. Nature communications.9, 1-14 (2018).

37. LeNoue, S. R. \& Riggs, P. D. Substance abuse prevention. Child and Adolescent Psychiatric Clinics.25, 297-305 (2016).

38. APA \& DSM-IV-TR Diagnostic and statistical manual of mental disorders, text revision. Washington, DC: American Psychiatric Association.75, 78-85 (2000). 
39. Torrens, M., Serrano, D., Astals, M., Perez-Dominguez, G. \& Martin-Santos, R. Diagnosing comorbid psychiatric disorders in substance abusers: validity of the Spanish versions of the Psychiatric Research Interview for Substance and Mental Disorders and the Structured Clinical Interview for DSM-IV. Am J Psychiatry.161, 1231-1237 https://doi.org/10.1176/appi.ajp.161.7.1231 (2004).

40. Hasin, D. et al. Diagnosis of comorbid psychiatric disorders in substance users assessed with the Psychiatric Research Interview for Substance and Mental Disorders for DSM-IV. Am J Psychiatry.163, 689-696 https://doi.org/10.1176/ajp.2006.163.4.689 (2006).

41. Beck, A., Steer, R. \& Brown, G. Manual for the Beck depression inventory-II 1996. San Antonio, TX: Psychological Corporation2 (1996).

42. Robins, L. N. et al. The Composite International Diagnostic Interview: an epidemiologic instrument suitable for use in conjunction with different diagnostic systems and in different cultures. Archives of general psychiatry.45, 1069-1077 (1988).

43. Romero-Sanchiz, P. et al. Plasma concentrations of oleoylethanolamide in a primary care sample of depressed patients are increased in those treated with selective serotonin reuptake inhibitor-type antidepressants. Neuropharmacology.149, 212-220 https://doi.org/10.1016/j.neuropharm.2019.02.026 (2019).

44. Koob, G. F. \& Le Moal, M. Drug abuse: hedonic homeostatic dysregulation. Science.278, 52-58 https://doi.org/10.1126/science.278.5335.52 (1997).

45. Diederich, K. et al. Synergetic effects of granulocyte-colony stimulating factor and cognitive training on spatial learning and survival of newborn hippocampal neurons. PLOS one4 (2009).

46. Torres-Rojas, C. \& Jones, B. C. Sex differences in neurotoxicogenetics. Frontiers in genetics.9, 196 (2018).

47. Ruiz, S. M. et al. Drinking history associations with regional white matter volumes in alcoholic men and women. Alcoholism: Clinical and Experimental Research.37, 110-122 (2013).

48. Vatsalya, V., Liaquat, B., Ghosh, H. \& Prakash Mokshagundam, K. S. \& J McClain, C. A review on the sex differences in organ and system pathology with alcohol drinking. Current drug abuse reviews.9, 87-92 (2016).

49. Lewis, B. \& Nixon, S. J. Characterizing gender differences in treatment seekers. Alcoholism: Clinical and Experimental Research.38, 275-284 (2014).

50. Wilsnack, R. W. et al. Gender differences in alcohol consumption and adverse drinking consequences: cross-cultural patterns. Addiction.95, 251-265 https://doi.org/10.1046/j.13600443.2000.95225112.x (2000).

51. Schwarz, J. M., Sholar, P. W. \& Bilbo, S. D. Sex differences in microglial colonization of the developing rat brain. Journal of neurochemistry.120, 948-963 (2012).

52. McCarthy, M. M., Nugent, B. M. \& Lenz, K. M. Neuroimmunology and neuroepigenetics in the establishment of sex differences in the brain. Nature Reviews Neuroscience.18, 471 (2017).

53. Wilhelm, C. J., Hashimoto, J. G., Roberts, M. L., Sonmez, M. K. \& Wiren, K. M. Understanding the addiction cycle: a complex biology with distinct contributions of genotype vs. sex at each stage. 
Neuroscience.279, 168-186 (2014).

54. Alfonso-Loeches, S., Pascual, M. \& Guerri, C. Gender differences in alcohol-induced neurotoxicity and brain damage. Toxicology.311, 27-34 (2013).

55. Oliveira, A., Rodríguez-Artalejo, F. \& Lopes, C. Alcohol intake and systemic markers of inflammationshape of the association according to sex and body mass index. Alcohol \& Alcoholism.45, 119-125 (2010).

56. Pedraz, M. et al. Sex differences in psychiatric comorbidity and plasma biomarkers for cocaine addiction in abstinent cocaine-addicted subjects in outpatient settings. Front Psychiatry.6, 17 https://doi.org/10.3389/fpsyt.2015.00017 (2015).

57. Flensborg-Madsen, T. et al. Comorbidity and temporal ordering of alcohol use disorders and other psychiatric disorders: results from a Danish register-based study. Compr. Psychiatr.50, 307-314 (2009).

58. Schneier, F. R. et al. Social anxiety disorder and alcohol use disorder co-morbidity in the National Epidemiologic Survey on Alcohol and Related Conditions. Psychological medicine.40, 977-988 (2010).

59. Conway, K. P., Compton, W., Stinson, F. S. \& Grant, B. F. Lifetime comorbidity of DSM-IV mood and anxiety disorders and specific drug use disorders: results from the National Epidemiologic Survey on Alcohol and Related Conditions. Journal of Clinical Psychiatry.67, 247-257 (2006).

60. Lewis, C. E., Bucholz, K. K., Spitznagel, E. \& Shayka, J. J. Effects of gender and comorbidity on problem drinking in a community sample. Alcoholism: Clinical and Experimental Research.20, 466476 (1996).

61. Garcia-Marchena, N. et al. Plasma concentrations of oleoylethanolamide and other acylethanolamides are altered in alcohol-dependent patients: effect of length of abstinence. Addict Biol.22, 1366-1377 https://doi.org/10.1111/adb.12408 (2017).

62. Westfall, N. C. \& Nemeroff, C. B. The preeminence of early life trauma as a risk factor for worsened long-term health outcomes in women. Current psychiatry reports.17, 90 (2015).

63. Schiepers, O. J., Wichers, M. C. \& Maes, M. Cytokines and major depression. Progress in NeuroPsychopharmacology and Biological Psychiatry.29, 201-217 (2005).

\section{Figures}


Figure 1

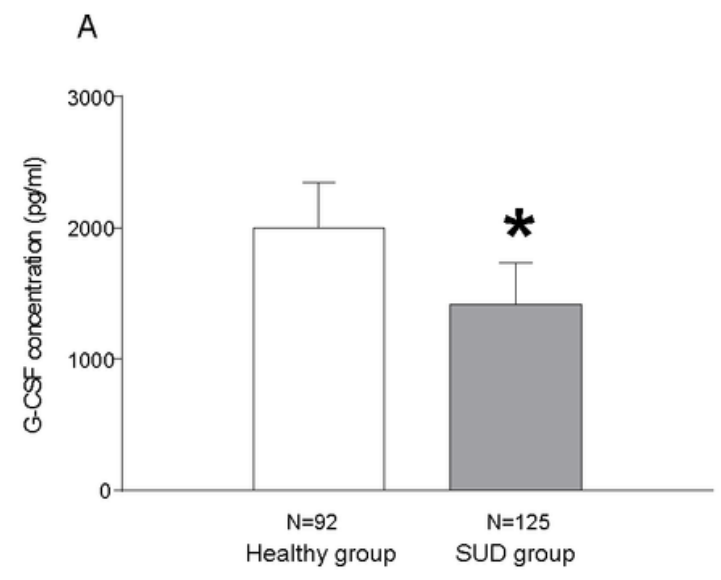

B

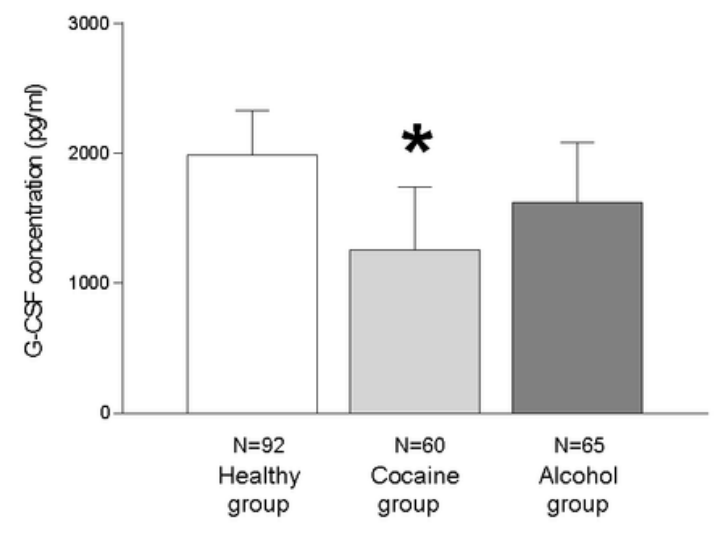

C

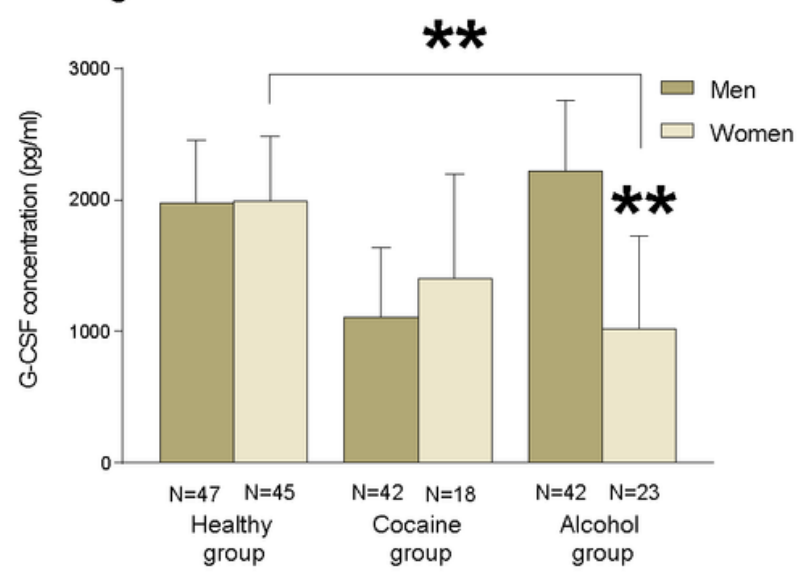

\section{Figure 1}

Plasma concentrations of G-CSF according to history of SUD. (A) Bars are estimated marginal means and confidence intervals (95\%) representing G-CSF (pg/ml) according to the SUD group.; (B) Bars are estimated marginal means and confidence intervals (95\%) representing G-CSF (pg/ml) according to the main substance in seeking treatment; $(C)$ Bars are estimated marginal means and confidence intervals (95\%) representing G-CSF ( $\mathrm{pg} / \mathrm{ml}$ ) according to the main substance in seeking treatment and according to the SUD group and sex. Data were analyzed by a two-way ANCOVA and * $p<0.05 * * p<0.01$ and $\star * * p<0.001$ and denote a significant main effect according to history of SUD and sex. 
Figure 2
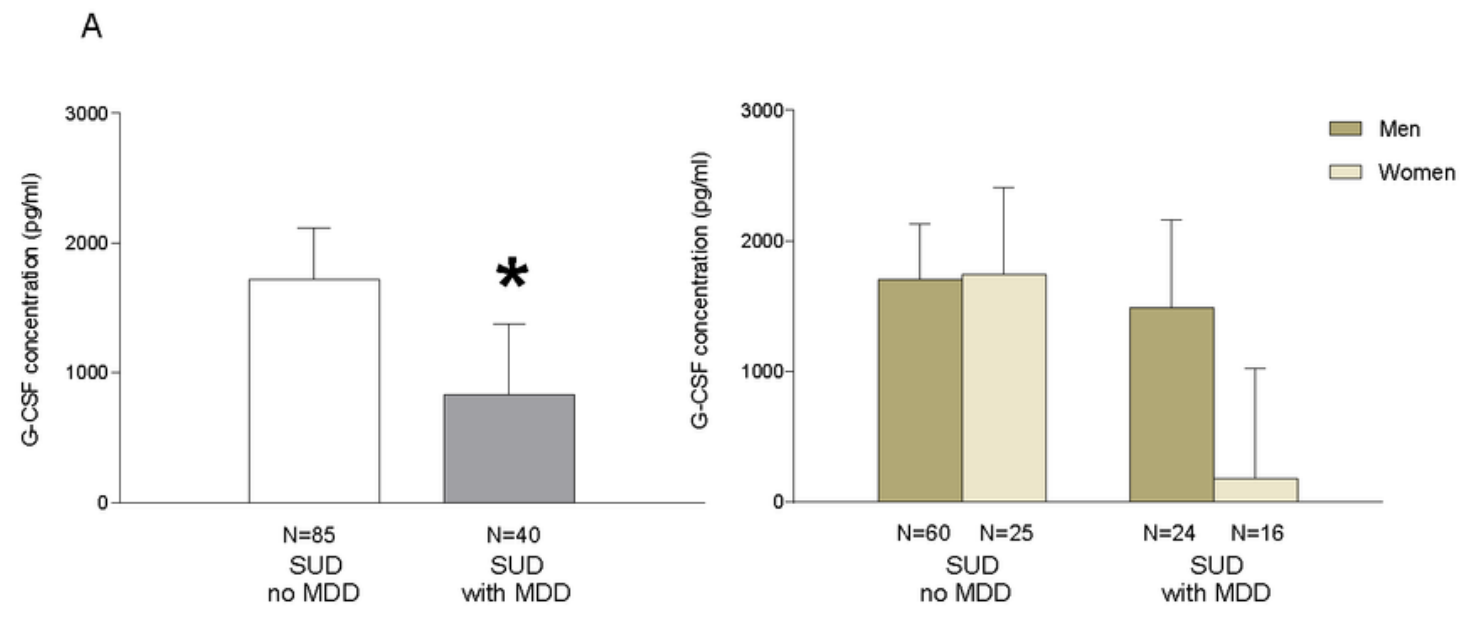

B
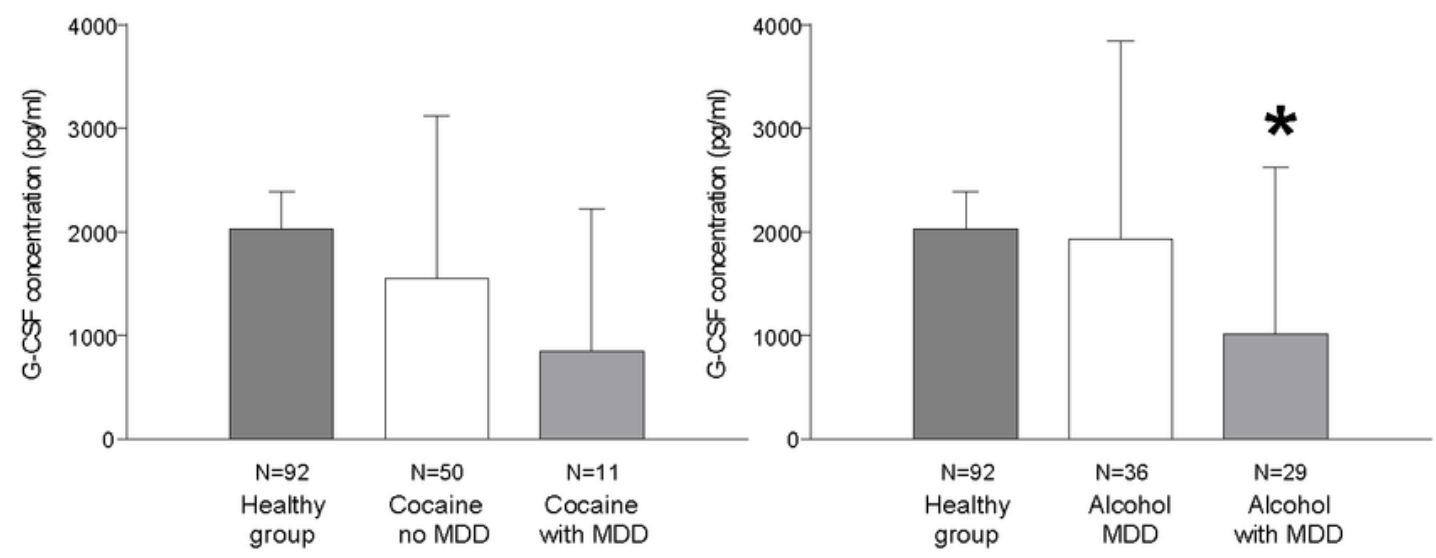

\section{Figure 2}

Plasma concentrations of G-CSF according to comorbid MDD in SUD. (A) Bars are estimated marginal means and confidence intervals (95\%) representing G-CSF (pg/ml) according to MDD comorbid disorders in the SUD group and according to the MDD comorbid disorders in the SUD group and sex; (B) Bars are estimated marginal means and confidence intervals (95\%) representing G-CSF (pg/ml) according to MDD 
comorbid disorders in the cocaine group and in the alcohol group. Data were analyzed by a one-way ANOVA and ${ }^{*}<0.05$ and denote a significant main effect according to comorbid MDD.

Figure 3

A

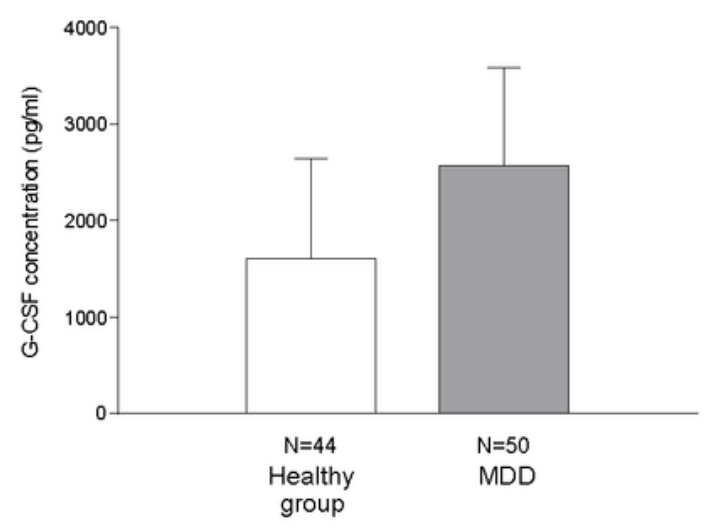

B
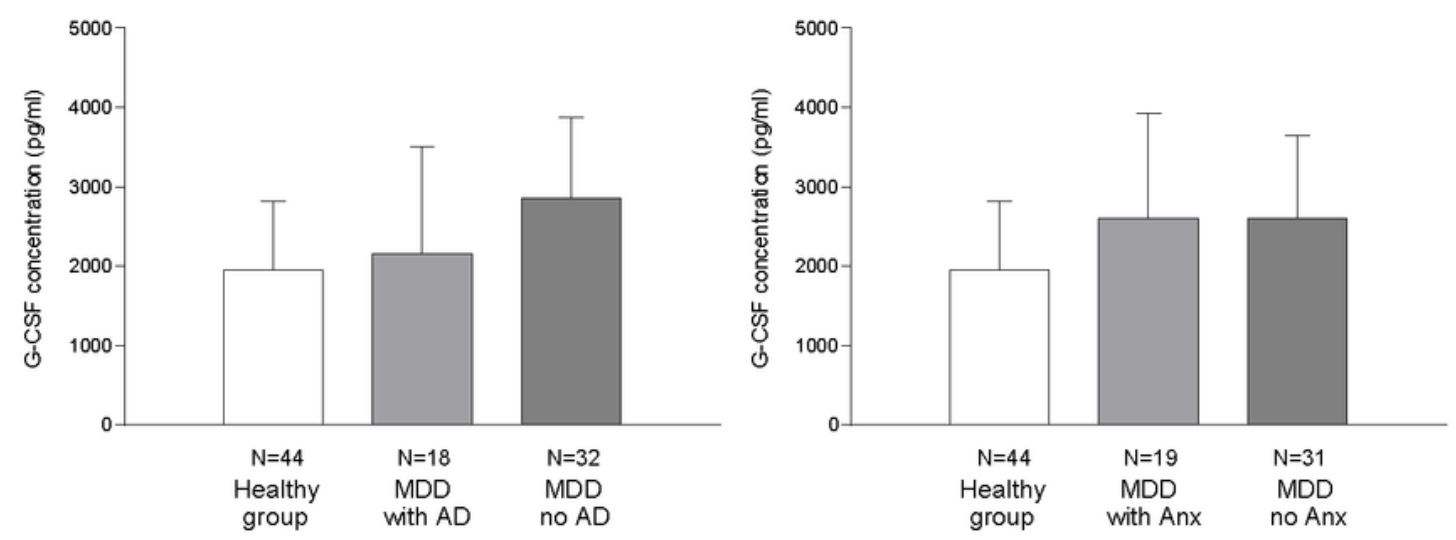

Figure 3

Plasma concentrations of G-CSF in alcohol study according to MDD. (A) Bars are estimated marginal means and confidence intervals (95\%) representing G-CSF ( $\mathrm{pg} / \mathrm{ml}$ ) according to MDD group; (B) Bars are estimated marginal means and confidence intervals (95\%) representing G-CSF ( $\mathrm{pg} / \mathrm{ml}$ ) according to 
Antidepressant medication in MDD group and according to anxiolytic medication in the MDD group. Data were analyzed by a one-way ANOVA and * $\mathrm{p}<0.05$ and denote a significant main effect.

\section{Supplementary Files}

This is a list of supplementary files associated with this preprint. Click to download.

- SupplemantaryInformation.docx 